\title{
A Case Report of Hepatic Lipidosis (HL) in Common Myna (Acridotheres tristis) Fed by High Caloric Broiler Diet in Iran
}

\author{
M. Hedayati1,2* M. Cheraghchi Bashi², S. M. Peighambari2 \\ ${ }^{1}$ Department of Animal Sciences, Faculty of Agricultural Sciences, Malayer University, Malayer, Iran \\ ${ }^{2}$ Department of Avian Diseases, Faculty of Veterinary Medicine, University of Tehran, Tehran, Iran \\ Email: ${ }^{*}$ mahvet77@gmail.com
}

Received 9 April 2014; revised 19 May 2014; accepted 29 June 2014

Copyright (C) 2014 by authors and OALib.

This work is licensed under the Creative Commons Attribution International License (CC BY). http://creativecommons.org/licenses/by/4.0/

(c) (i) Open Access

\begin{abstract}
Hepatic lipidosis (HL) is common in birds fed all seed, high caloric or high-fat diets, especially psittacine birds. The most common cause of avian HL is obesity related to over feeding. This case report describes the clinical and pathological features of 10 Common Myna (Acridotheres tristis) fed by commercial broiler diet and were presented for depression and anorexia, with severe hepatopathy associated hepatic lipidosis (HL). The patients died 4 days after presentation, and severe hepatomegaly was observed at necropsy. Based on histopathological findings, the acute hepatic lipidosis (HL) was found.
\end{abstract}

Keywords

Common Myna, Diet, Hepatic Lipidosis (HL), Iran

Subject Areas: Animal Behavior, Veterinary Medicine

\section{Introduction}

Myna is one of the most companion birds in Iran. The lack of knowledge about nutrition for this bird with their owners and miss-feeding with high caloric diet which are not specific for pet birds and inappropriate cages with not enough space for bird movement are reasons to cause metabolic disease in this type of bird. The most notable metabolic disease in myna in Iran is found to be hepatic lipidosis.

\section{Case History}

In this case, 10 Common Myna (Acridotheres tristis) were fed by broiler diet and presented with depression,

\footnotetext{
${ }^{*}$ Corresponding author.
}

How to cite this paper: Hedayati, M., Cheraghchi Bashi, M. and Peighambari, S.M. (2014) A Case Report of Hepatic Lipidosis $(\mathrm{HL})$ in Common Myna (Acridotheres tristis) Fed by High Caloric Broiler Diet in Iran. Open Access Library Journal, 1: e476. http://dx.doi.org/10.4236/oalib.1100476 
anorexia and respiratory distress signs to veterinary clinic of Tehran university in Iran. High energy in this seed was about $3100 \mathrm{Kcal} / \mathrm{kg}$ of seed, and these cages could not provide enough space for birds to freely movement. On physical examination, body condition scoring was increased about 4. In palpation of body not uniformity, and radiographic examination of patients was used. On radiographs, hepatomegaly or distress in bone were not seen. Primary treatment for these birds consider of clinical signs with B-complex syrup and correction of feeding by vegetables and fruits then 4 days after treatment all of birds were died and in necropsy, gross findings included excessive subcutaneous fat deposits and severe hepatomegaly around organs were noticed. The liver was enlarged, gold-brown, and friable. Collected liver tissue was fixed in 10\% formalin: paraffin embedded, sectioned, and stained with $\mathrm{H} \& \mathrm{E}$ method for light microscopic examination. On the microscopical observation (Figure 1), the hepatocytes were swollen with variable sized clear vacuoles that pushed the nuclei peripherally which were due to fatty change of hepatocytes. However, pykonosis of hepatocytes was not prominent. It suggested there was no hepatic necrosis. The histopathological findings were highly suggestive of a severe hepatopathy resulting from HL.

\section{Discussion}

Hepatic lipidosis or fatty liver disease is one of the gradually disease in avian species. There was saw the number of birds are very ill, life-threateningly so, or possibly having died suddenly. Often the owners lack of knowledge of risk of feeding with the seeds, peanuts or other oily foods or highly high caloric foods to the birds which they obviously loves to eat will lead to a very serious problem for them. These are truly cases of "loving your bird to death". Any bird can fall victim to fatty liver disease. Most commonly cockatiel, lovebird, mynah, and Amazons are diagnosed with this silent killer. However, ascites is rarely seen in birds with hepatopathy and is more associated with concurrent cardiac disease [1]. The diseased birds can die without premonitory, or signs can be nonspecific [1]. Birds that are on all seed diets or are being fed high energy diets and receiving little exercise or not had enough movement are prone to fatty livers. Pets dealing with this disease may show a variety of signs including, but not limited to oily feathers, feather picking, decreased exercise tolerance, obesity, diarrhea, ataxia (wobbliness), anorexia-complete/sudden, poor growth and reduced disease resistance, sudden illness or death. When these birds presented to veterinarian clinic, these signs along with palpation findings the enlarged abdomens where the edges of the liver may actually be felt and sometimes yellow urates are noted. Further diagnostics are usually recommended and may include serum analysis, radiographs (x-rays), ultrasounds and/or biopsies. Fat accumulation in the liver is caused by increased fatty acid production due to the high fat diet. These fatty acids are not immediately being used, so they get stored in the liver. Once there, they interfere with the liver's function. Important nutrients such as calcium suffer impaired absorption and, as sequelae of both diet



Figure 1. Microscopic assay in hepatocyte and fatty change in hepatocytes. 
and disease vitamin/mineral deficiencies exist. This problem does not occur overnight and, likewise, the return to health is not immediate. The basis of treatment is elimination of fat in the diet replaced by healthy foods such as pellets, fresh vegetables, and fresh fruits. Any bird suffering from fatty liver may need supportive care such as fluids, medications to decrease blood ammonia levels, and treatment of secondary infections.

Diagnostics of HL in companion birds the first procedure done physical examination, an enlarged liver can be visualized by moisturing the skin over the liver as it extends beyond the caudal border of the sternum [2]. An enlarged liver will displace other organs in the coelomic cavity and this may be recognized on palpation and radiographically, the margins of the liver should not extend past the sternum on the lateral view [2]. With enlargement there will be a dorsal deflection of the proventriculus and caudodorsal displacement of ventriculus (to level of acetabulum) [2]. There is a loss of the hourglass cardiac-hepatic silhouette on the dorsoventral view and the liver will extend beyond an imaginary line drawn from the scapula to acetabulum [2]. Contrast studies are useful in determining the extent of liver changes, as ascites or an enlarged fluid-filled proventriculus can produce the appearance of hepatomegaly. Radiographs are indicated before endoscopic or surgical evaluations are considered to rule-out concurrent disease processes and determine the size and location of the liver. Ultrasound can be a useful diagnostic tool in many liver diseases. Even the livers in birds as small as budgerigars can be imaged with a small probe (7.5 MHz finger probe). The air sacs are avoided by keeping the probe on the ventral midline along the caudal rim of the sternum. Ultrasound-guided fine needle aspirates can be considered for birds presenting as anesthetic risks. Elevations in serum analysis aspartate amino transferase (AST, SGOT) and lactate dehydrogenase (LDH) can occur in liver disease, but are not specific for the liver [1] [3]. The best use of these tests is to evaluate them in combination with the results of other more specific tests, such as creatinine kinase (CK), a muscle specific enzyme, to exclude muscle damage as a cause of the elevations [4]. SGOT is found in kidney, spleen, heart, skeletal muscle, small intestine, lung, brain, as well as the liver [1] [3]. The most common causes of serum elevation are with liver disease and muscle damage [3]. Elevations of this enzyme have been reported with vitamin $\mathrm{E}$ and selenium deficiencies, and intoxication from pesticides and carbon tetrachlorides [3]. Lactic dehydrogenase (LDH) is a highly labile enzyme that rises and falls more quickly than AST [1] [3]. Large amounts of this enzyme are found in myocardium, kidney, liver, and muscle [4]. Hemolysis of the serum and hepatic diseases in psittacines can result in elevated levels of LDH [3]. Serum bile acid measurement is a liver function test because extraction, conjugation, and secretion of bile acids are all functions of the liver [1] [5]. When liver function is impaired, bile acids are not properly reabsorbed from the blood and the proportion of excreted bile acids reaching the peripheral circulation increases [3] [6]. Elevations have correlated well with liver disease in many avian species [3] [4]. It is recommended to submit a single, fasted (3 to 4 hours) sample for bile acids determinations in birds [4] [6]. Bile acids are labile in plasma and samples should be run within 48 to 72 hours of collection. Cholesterol is a major lipid that is a precursor of all steroid hormones and bile acids. The source is animal proteins in the diet and it is synthesized in the liver. Increased serum levels can be associated with high fat diets, hypothyroidism, liver disease, starvation, budgies with xanthomatosis, obese birds, and very high levels usually accompany lipemia, especially in Amazons [3]. Decreases in albumin can be a non-specific indicator of chronic liver disease, as albumin is manufactured by the liver [3]. This decrease will occur very late in the course of the disease [3]. A yellow appearance of the avian plasma or serum should not be mistaken for jaundice, as birds do not produce appreciable amounts of bilirubin. The yellow color is suspected to be due to carotene pigments from the diet. Most chronic diseases, including hepatic diseases can induce a depression anemia. The definitive diagnosis of liver disease is obtained by a liver biopsy and histopathology [2] [3]. This not only describes the pathological process, but also provides information about the degree of change, which is important for prognosis. The liver can be biopsied from three approaches; percutaneous, endoscopic, and by laparotomy [3]. With suspected liver disease, pre treatment with vitamin $\mathrm{K}$ is warranted. The liver is the site of vitamin K dependent coagulation factor synthesis [3]. Excessive hemorrhage can accompany chronic hepatic diseases [3]. The indications for a biopsy are persistently elevated liver enzymes, radiographic changes, and a limited or absent response to therapy. General therapy although the best treatment plan should include a specific therapy directed at the causative agent, this is frequently not possible. In many liver diseases the specific etiology is unknown, or is an agent that is nonresponsive to current treatments (viruses, amyloid), or is no longer present (toxins). Supportive care is important, especially with acutely ill birds. If the bird can be supported through an acute episode and then maintained on a regimen designed to minimize liver insults, the bird can enjoy varying intervals of good health. Oxygen therapy is helpful for birds with anemia, ascites, and hepatomegaly which results in respiratory compromise. Excessive ascitic fluid can be aspirated to provide immediate respira- 
Table 1. Ingredients of diets (DANE PARS ${ }^{\circledR}$ company of seed in IRAN).

\begin{tabular}{cc}
\hline Energy & $3100 \mathrm{kcal} . / \mathrm{kg}$ \\
Protein & $21 \%$ \\
Calcium & $0.9 \%$ \\
\hline
\end{tabular}

tory relief as long as appropriate precautions are taken to prevent severe albumin depletion and hypovolemia. Diuretics such as furosemide can be used to control ascites. Fluid therapy is important as many of these birds are not eating or drinking enough to meet their needs. With severely compromised birds, subcutaneous fluids may be safer initially, until a catheter (IV or IO) can be placed if needed. For hepatic diseases, an easily digested, low-residue diet is recommended. Vitamin supplementation is also important. Lactulosec given orally changes the intestinal $\mathrm{pH}$ and prevents the absorption of ammonia [3]. Select disease conditions metabolic disorders of the liver include: hepatic lipidosis [1] [3]. In Indian hill mynahs, a viral or metabolic abnormality is suspected [5]. Fatty liver disease: Fatty liver disease (hepatic lipidosis) is an insidious condition that is poorly understood. It is a common hepatic lesion seen in budgies, cockatiels, and Amazons [2]. The typical presentation is an acutely ill bird. The clinical signs include sudden and complete anorexia, depression, dyspnea, and ataxia [2]. Commonly the birds are obese and most have been on all-seed diets for the major part of their lives [3]. The liver is usually palpably enlarged and yellow in color. The probable pathogenesis is by an excessive lipid accumulation in the liver caused by fatty acid synthesis exceeding their utilization [1]. Some possible etiologies include endocrine diseases (hypothyrodism or diabetes mellitus), any disease condition interfering with nutrient digestion or absorption, or a primary defect in fat metabolism [1]. Often these birds will have elevated bile acids and commonly the other liver enzymes may be normal. A liver biopsy is important for prognosis. Therapy is supportive [3]. According to a recent report [1], it is suggested that this condition may be genetically predisposed because of a species-oriented physiology. Some other possible causes are stress, which is related to disease exposure (immunologic stress), crowding, and nutritional stress related to periodic starvation by diet changes [1]. This patients were fed DANE PARS ${ }^{\circledR}$ company seed that highly energy and proteins (Table 1), Fruits and vegetables were some supplied. In this seed, high level carbohydrate diets may serve to increase the accumulation of fat in hepatocytes [6] and this cases not enough space to movement that severe occurrence of HL. HL results from increased fatty acids to the liver caused by high-fat diet, dietary deficiency of lipotropic factors such as choline, biotin, methionine, diabetes mellitus, thyroid dysfunction, toxicosis, and steroid administration [1]. Accordingly, overfeeding of a high caloric diet caused the bird's obesity and thus induced HL. Because of this seed designed to broiler chicken with $3200 \mathrm{kcal}$ per 1 kilogram of feed and energy requirement in mynah bird was $2000 \mathrm{kcal}$ per $1 \mathrm{~kg}$ of feed [7].

The recommended Diet of myna: 100-gram bird requires $25 \mathrm{kcal}$ daily to meet maintenance energy requirements, depending on activity level and any additional energy demands (such as cold temperatures). An example of a diet that meets known nutrient needs for one bird includes: 25 grams proprietary soft bill pellet (such as one of the above mentioned brands) 15 grams of diced fruit, 7 grams of mixed vegetables, 5 grams of minced leafy greens [8].

\section{Acknowledgements}

This study was done in central laboratory of Faculty of Veterinary Medicine, University of Tehran, Tehran, Iran.

\section{References}

[1] Cranfield, M.R., Shaw, M., Beall, F. and Skjoldager, M. (1990) A Review and Update of Avian Malaria in the African Penguin (Speniscus demersus). American Association of Zoo Veterinarians, 243-248.

[2] Gould, W.J. (1992) Liver Disease in Psittacines. In: Miller, R.E., Ed., Current Veterinary Therapy XI Small Animal Practice. W.B. Saunders, Philadelphia, 1145-1150.

[3] Hochleithner, M. (2002) Biochemistries. In: Ritchie, B.W, Harrison G.J. and Harrison, L., Eds., Avian Medicine: Principles and Application, Wingers Publishing Inc., Lake Worth, 223-245.

[4] Lumeij, J.T. (1994) Avian Clinical Enzymology. Seminars in Avian \& Exotic Pet Medicine, 3, 14-24.

[5] Hoefer, H.L. (1997) Diseases of the Gastrointestinal Tract. In: Altman, R.B., Clubb, S.L. and Dorrestein, G.M., Avian 
Medicine and Surgery, Saunders, Philadelphia, 419-453.

[6] Hoefer, H.L. (1994) Bile Acid Testing in Psittacine Birds. In: Fudge, A.M., Ed., Seminars in Avian \& Exotic Pet Medicine, 2, 14-24.

[7] Mary, I.P. (1994) Nutrient Requirements of Poultry. 9th Revised Edition, National Academies Press, Washington DC.

[8] Dierenfeld, E.S. (1995) Nutrition Husbandry Guidelines for the Bali Mynah (Leucopsar rothschildi) Species Survival Plan. American Association of Zoos and Aquariums. 